\title{
Extended Calibration Technique of a Four-Hole Probe for Three-Dimensional Flow Measurements
}

\author{
Suresh Munivenkatareddy and Nekkanti Sitaram \\ Thermal Turbomachines Laboratory, Department of Mechanical Engineering, Indian Institute of Technology Madras, \\ Chennai 600 036, India
}

Correspondence should be addressed to Suresh Munivenkatareddy; suremreddy@gmail.com

Received 9 December 2015; Revised 2 April 2016; Accepted 25 May 2016

Academic Editor: Awatef Hamed

Copyright (C) 2016 S. Munivenkatareddy and N. Sitaram. This is an open access article distributed under the Creative Commons Attribution License, which permits unrestricted use, distribution, and reproduction in any medium, provided the original work is properly cited.

\begin{abstract}
The present paper reports the development and nonnulling calibration technique to calibrate a cantilever type cylindrical fourhole probe of $2.54 \mathrm{~mm}$ diameter to measure three-dimensional flows. The probe is calibrated at a probe Reynolds number of 9525 . The probe operative angular range is extended using a zonal method by dividing into three zones, namely, center, left, and right zone. Different calibration coefficients are defined for each zone. The attainable angular range achieved using the zonal method is \pm 60 degrees in the yaw plane and -50 to +30 degrees in the pitch plane. Sensitivity analysis of all the four calibration coefficients shows that probe pitch sensitivity is lower than the yaw sensitivity in the center zone, and extended left and right zones have lower sensitivity than the center zone. In addition, errors due to the data reduction program for the probe are presented. The errors are found to be reasonably small in all the three zones. However, the errors in the extended left and right zones have slightly larger magnitudes compared to those in the center zone.
\end{abstract}

\section{Introduction}

Turbomachinery flows are highly unsteady and three dimensional. The key to further improvement in turbomachinery is through understanding the three-dimensional flow through their components such as rotors and stators. Such three dimensional flows encountered in turbomachines can be analyzed by flow visualization, computational methods, and direct measurements of the flow field. However, flow visualization has limitations since the techniques serve only to the locate regions of interest in the flow, and computational methods are expensive and are not fully reliable. Only direct measurement of the flow can provide quantitative data of flow parameters, such as total and static pressures, velocities and Mach numbers, and flow angles to understand the flow better. Pressure probes are one of the options to measure the flow parameters directly by inserting them into the flow field of turbomachines [1]. The prominent advantages of pressure probe techniques over hot-wire probes and optical techniques are their ability to measure pressure within the flow, robustness, simplicity, and cost effectiveness.

Since Henri Pitot used a simple bent tube to measure the total pressure in fluid flow in 1732, a broad variety of pneumatic probes have been developed over years. Recently, Telionis et al. [2] have made a comprehensive survey of multihole pressure probes for flow measurements. Depending on the velocity range, angular range required, and types of turbomachinery, specific probe head geometries have been designed. All these probes can determine flow quantities such as total and static pressure, flow angles, or Mach numbers by measuring the pressures at different locations on the probe head. The minimum number of pressure holes on the pressure probe depends on the dimensionality of the flow field measured. A simple pressure probe used to measure two-dimensional flows has three pressure holes [3] while the minimum number of holes to measure threedimensional flows is four. Multihole pressure probes of four [4], five [5], seven [6], and more holes strategically placed on 
aerodynamic bodies such as a sphere, hemisphere, and prism have been used successfully to measure three-dimensional flows. In principle, a four-hole probe can measure the four quantities that are required to completely define the flow. However, for the sake of symmetry in both yaw and pitch planes, five-hole probes are usually employed. When the yaw and pitch angles of the flow exceed the usual operating range of five-hole probes, seven-hole probes or probes with a larger number of holes are employed. Usually, the operating angular range of three-, four-, and five-hole probes is limited to \pm 30 degrees, while the operating range of probes with perpendicular holes is limited to \pm 40 degrees, according to Pisasale and Ahmed [7]. The limitation is due to the value of the denominator, $D$, in the definition of the calibration coefficients, becoming very small, zero, or negative, when the yaw or pitch angle exceeds a certain value. In such case, the calibration coefficients become very large, or singular, or changes sign.

Ostowari and Wentz Jr. [8] suggested a method to increase the operative range of a five-hole probe using a nulling method and an operative range of \pm 85 degrees was achieved. However, nulling is not always possible, especially in turbomachinery flows where large data are to be acquired. This limitation in operative angular range of multihole probes can be solved by discriminating the angular range into a number of zones. Zonal methods [9] to increase the calibration range of the multihole probe have evolved over recent years, as it is a simple technique to achieve a wide operative angular range of pressure probes. In this method, the operating range is divided into a number of zones based on the maximum probe hole pressure reading and unique calibration coefficients are defined for each zone. Using this method, the calibration range of seven-hole probes [10] has been increased as high as \pm 80 degrees in nonnulling mode. Recently Argüelles Díaz et al. [11] have carried out a mathematical analysis of the limits of operating range and data reduction techniques, for increasing the angular range of three-hole cylindrical probes and the operating angular range was increased to \pm 70 degrees from \pm 35 degrees. They also used a zonal method to increase the angular range of a three-hole cobra type pressure probe [12] and attained an angular range of \pm 105 degrees from the typical \pm 30 degrees.

As previously stated, a multihole probe having four pressure holes can be used to measure three-dimensional flows. The size of the five-hole probe, seven-hole probe, and probe of higher number of holes is larger, causing larger velocity gradient and blockage errors. Shepherd [13] has reported a four-hole probe for measuring three-dimensional flows and attained an angular range of \pm 45 degrees in both yaw and pitch angles by discriminating the angular range into six different zones. Discriminating into a large number of zones and using different data reduction for each zone is again a tedious process. So a four-hole probe able to measure threedimensional flows where the flow parameters vary widely in both magnitude and direction with a minimum number of zone divisions is preferable. Recently, Schlienger [14] developed a miniature four-hole probe of $1.2 \mathrm{~mm}$ diameter made out of brass. His design uses a probe head with an elliptical shape on top of the probe tip that has an aspect ratio of $2: 1$. The probe was calibrated over an angular range of \pm 20 degrees in the yaw plane and \pm 16 degrees in the pitch plane and was found to be reasonably accurate when compared to a five-hole probe. This type of probe is found to be very useful to measure the flows in diffusers of centrifugal compressors and in labyrinth seals of axial turbines, where the spanwise flow angles are usually small. However, for any turbomachinery flow measurements, pressure probes with very small measurement volumes and the capability to measure large flow angles in both the yaw and pitch planes are needed.

\section{Objective}

The objective of the present work is to develop a miniature four-hole probe with a hemispherical shape on top of the probe tip, as opposed to an elliptical shape [14], and calibrate the probe in nonnulling mode to achieve a large operative angular range with the use of a minimal number of zones, with acceptable accuracy.

\section{Probe Design and Fabrication}

The four-hole probe used in the present investigation is fabricated using stainless steel tube of $2.54 \mathrm{~mm}$ diameter to which a probe head of $2.54 \mathrm{~mm}$ diameter and $8 \mathrm{~mm}$ length is silver brazed. Figure 1 shows the three orthogonal views of the probe head. The probe head is made in a hemispherical shape on the top of the probe tip. Four pressure holes of $0.3 \mathrm{~mm}$ are drilled on the hemispherical surface of the probe head. Three $0.3 \mathrm{~mm}$ pressure holes $\left(P_{1}, P_{2}\right.$, and $P_{3}$ in Figure 1) are drilled in the yaw plane at a construction angle, $\delta_{1}$, of 50 degrees apart, similar to a three-hole probe used to measure two-dimensional flows. The fourth hole ( $P_{4}$ in Figure 1) is drilled at a construction angle, $\delta_{2}$, of 45 degrees in the pitch plane on the hemispherical surface of the probe head. The three pressure holes in the yaw plane are connected to $0.45 \mathrm{~mm}$ drilled holes in the head which are parallel to the probe axis while the fourth hole is connected to a drilled hole of $1 \mathrm{~mm}$ diameter. Three tubes of $0.45 \mathrm{~mm}$ and one tube of $1 \mathrm{~mm}$ diameter are inserted into 0.45 and $1 \mathrm{~mm}$ drilled holes in the head and are silver brazed. The stem diameter is further increased to $6.35 \mathrm{~mm}$ using a transition piece. For faster response of the pressures, tubes of 0.45 and $1 \mathrm{~mm}$ diameter are inserted into $1.5 \mathrm{~mm}$ diameter tubes in the transition region and are silver brazed. The total length of the probe is about $570 \mathrm{~mm}$. A schematic of the complete probe is shown besides the orthogonal views of the probe head. As seen from the figure, the probe body is perpendicular to the flow, which can lead to crossflow effects. The probe configuration is selected for use in a centrifugal fan, where space is limited. An elliptic body is desirable but difficult to manufacture. The pressure distributions presented in Figure 4 seem to be satisfactory without showing any crossflow effects. The probe head is made as small as possible in order to minimize flow blockage and disturbance within the limitations of manufacturing constraints. The probe is ideally suited to measure three-dimensional flows in confined spaces such as diffusers of centrifugal fans and labyrinth seals. 


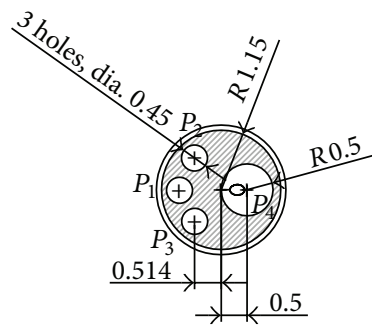

Section B-B

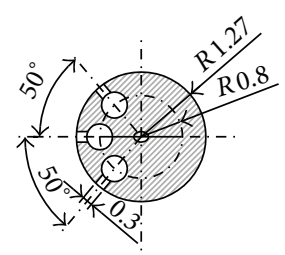

Section A-A

Four-hole probe head (2.54 mm, 0.1" Dia.)

Material: stainless steel All dimensions in $\mathrm{mm}$ Scale $10: 1$

Probe head
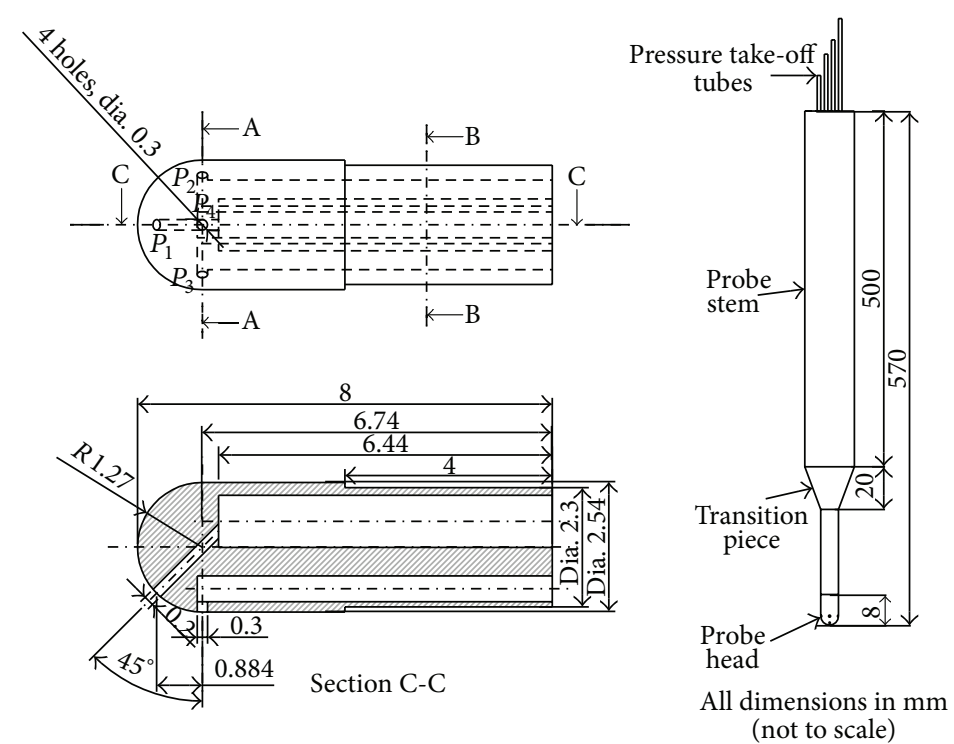

FIGURE 1: Orthogonal views of the four-hole pressure probe head and schematic of the probe.

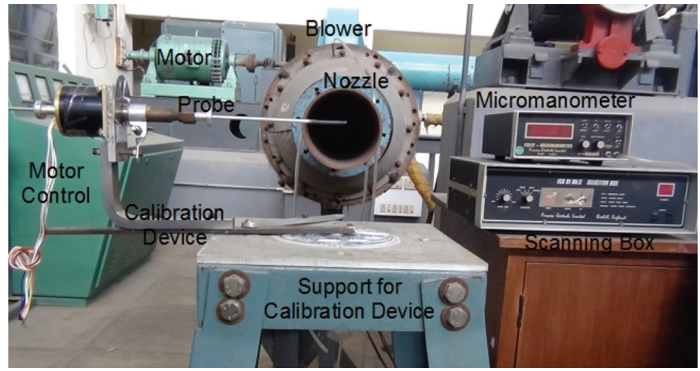

FIGURE 2: Calibration tunnel, calibration device, probe, and instrumentation.

In such applications, using five-hole probe, seven-hole probe, or probe of higher number of holes is difficult and results in large errors due to blockage. The present four-hole pressure probe has relatively high spatial resolution; that is, center-tocenter distance between holes in the pitch plane is $0.88 \mathrm{~mm}$ and center-to-center distance in the yaw plane is $1.95 \mathrm{~mm}$.

\section{Calibration Tunnel, Calibration Device, Instrumentation, Calibration Procedure, and Calibration Program}

The four-hole probe is calibrated in an open-jet, low speed calibration tunnel facility of Thermal Turbomachines Laboratory, Department of Mechanical Engineering, IIT, Madras, which is shown in Figure 2. The calibration tunnel consists of a low pressure centrifugal fan driven by a variable speed DC motor. The high pressure nonuniform turbulent delivery air from the centrifugal fan is sent through a settling chamber to the jet exit through a contraction section of $9: 1$ contraction ratio. The flow in the jet of the calibration tunnel is uniform within $\pm 0.5 \%$ of the center line stream velocity and the turbulence level is about $1 \%$. The flow in the core region of the jet is found to be along its axis without any deviation. The total pressure is measured from the averaged wall static pressures on the settling chamber wall. The static pressure is taken as atmospheric. Earlier quantification tests on the calibration tunnel using a three-hole probe at the nozzle exit verified that the total pressure measured from the averaged wall static pressures on the settling chamber wall is equal to the total pressure within $\pm 1 \%$ and the static pressure is atmospheric. This calibration tunnel is routinely used for the calibration of single and multihole pressure probes and single and multisensor thermal anemometer probes.

The probe is mounted in a calibration device. The calibration device consists of a base plate, a c-clamp, and protractors with pointers for measurement of the pitch $(\beta)$ and yaw $(\alpha)$ angles. The twenty-channel single selection scanning box (model number FCO 91-3) and FC012 digital micromanometer with a range of 1-200 $\mathrm{mm}$ of water and sensitivity of $0.1 \mathrm{~mm}$ of differential air pressure, manufactured by Furness Control Ltd., Bexhill, London, were used to measure probe pressures. The micromanometer uses the output signals from the selection box to obtain the pressure readings.

The four-hole probe is calibrated at a Reynolds number of 9525 ( $60 \mathrm{~m} / \mathrm{s}$ velocity), based on the probe head diameter. A total of $425(25 \times 17$ in the yaw and pitch planes, resp.) of calibration points were obtained over a yaw angle range of \pm 60 degrees and a pitch angle range of 30 to -50 degrees, at an interval of 5 degrees in the yaw and pitch angle range.

\section{Results and Discussion}

5.1. Calibration Coefficients and Curves. The pressure data recorded during calibration was used to plot calibration 
curves using the traditional calibration coefficients defined for one such probe earlier [14].

The traditional normalized calibration coefficients for the four-hole probe are defined as follows:

$$
\begin{aligned}
C_{\text {PYAL }} & =\frac{P_{2}-P_{3}}{D}, \\
C_{\text {PPITCH }} & =\frac{P_{1}-P_{4}}{D}, \\
C_{\text {PTOTAL }} & =\frac{P_{O}-P_{1}}{Q}=\frac{P_{O}-P_{1}}{P_{O}}, \\
C_{\text {PSTATIC }} & =\frac{\left(P_{2}+P_{3}\right) / 2-P_{S}}{Q}=\frac{\left(P_{2}+P_{3}\right) / 2}{P_{O}},
\end{aligned}
$$

where $D=P_{1}-\left(P_{2}+P_{3}\right) / 2, C_{\text {PYAW }}$ represents yaw coefficient, $C_{\text {PPITCH }}$ represents pitch coefficient, $C_{\text {PTOTAL }}$ and $C_{\text {PSTATIC }}$ are the total and static pressure coefficients, and $D$ is the normalization factor that is introduced in all the coefficients. These coefficients become independent of dynamic pressure when normalized with the factor $D$. As a result, $C_{\text {PYAW }}$ and $C_{\text {PPITCH }}$ are only a function of flow angles and both total and static pressure coefficients provide the total and static pressures of the flow.

The calibration curve $C_{\text {PYAW }}$ versus $C_{\text {PPITCH }}$ for the probe plotted at an interval of 10 degrees in both the yaw and pitch planes using the traditional calibration coefficient definition is shown in Figure 3. From this curve, it is clearly evident that the probe cannot be used to measure the yaw angle beyond \pm 30 degrees. This limitation in angular range of the probe using the traditional coefficients is due to the arising of singular points beyond \pm 30 degrees in yaw angle. For a typical cylindrical probe using the above angular coefficients, singular points appear approximately at \pm 37 degrees. Singular points in the angular coefficients appear when the normalized factor $D$ becomes zero and for the present probe these points appear somewhere in between \pm 35 degrees and \pm 40 degrees in yaw angle. However, the problem of singularity in the angular coefficients is not the real hurdle to increase the angular range of the probe. The real limitation to the angular range occurs when double points appear. Double points appear when the equations used for angular coefficients produce dual solutions [12]. Singularities in angular coefficients can be avoided by discriminating several zones within the whole angular range and defining a new normalizing factor $D$ in each zone, such that the normalized calibration coefficients in each zone are independent of both static pressure and dynamic pressure; and the normalizing factor $D$ does not become zero in their respective zones. The other way to avoid this singularity is by using the real dynamic pressure, as the normalizing factor [15], but in such a method, an iterative procedure is required to arrive at the correct value of dynamic pressure.

The calibration coefficients and normalizing factor are calculated using the pressures measured by the four holes of the probe. Hence, it is necessary to analyze the pressure data of each hole of the probe at various flow angles for discrimination of zones based on their behavior. In this
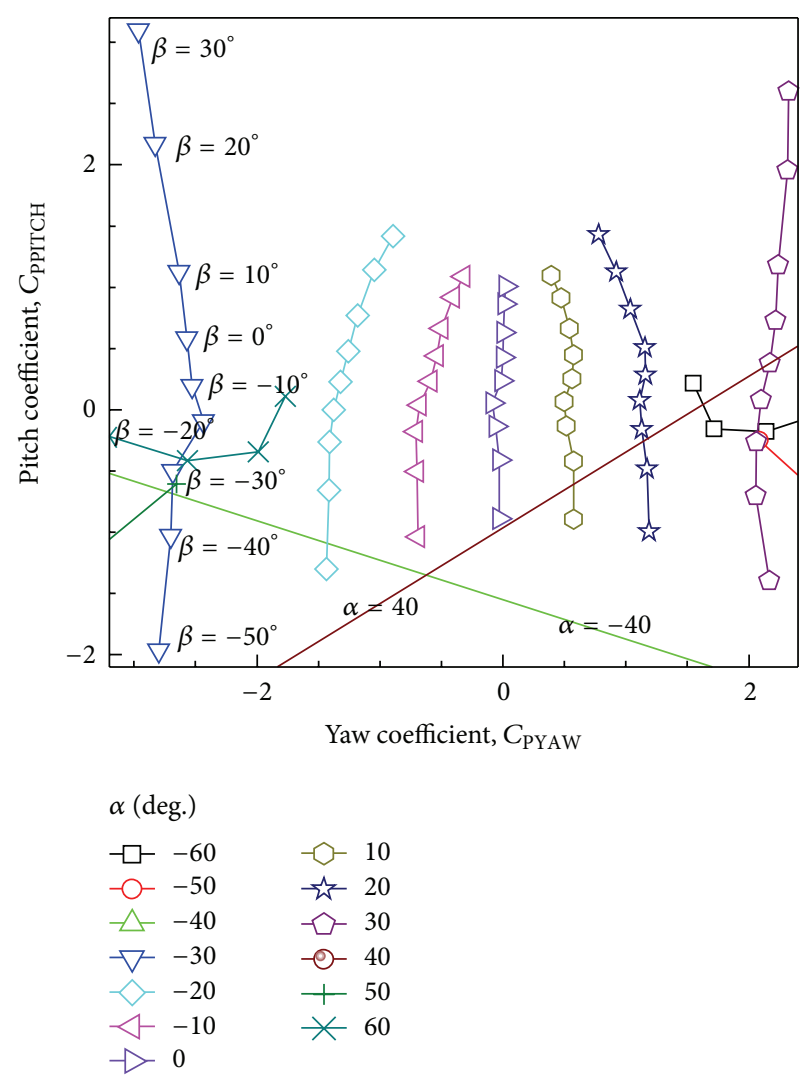

FIgURE 3: $C_{\text {PPITCH }}$ versus $C_{\text {PYAW }}$ calibration curve using traditional calibration coefficients.

section, an effort has been made to analyze the pressure coefficient distributions of the four probe holes over an angular range of \pm 60 degrees in yaw and +30 to -50 degrees in pitch. Figure 4 shows the distributions of pressure coefficient as a function of yaw angle for three values of pitch angles of $0,+30$ (maximum value) and -50 (minimum value) degrees. They are normalized with the measured total pressure $\left(P_{O}\right)$ read during calibration, that is, $p_{i}=\left(P_{i} / P_{O}\right)$. The pressure coefficient of the respective hole is expected to be maximum $\left(p_{i}=1\right)$ when the flow is aligned with the hole axis. And it is expected to be minimum when the flow is perpendicular to the hole axis. For a typical cylindrical three-hole probe used in measuring 2D flows, these pressure coefficients are maximum at 0 degrees for center hole and maximum for the left and right holes at their respective tap angles (construction angle, $\delta_{1}$ ) when the pitch angle is zero. The minimum and maximum pressures for the two side holes, $p_{2}$ and $p_{3}$, are also presented in Figures 4(a)-4(c). These are shown by a starred symbol, with $\mathrm{L}$ and $\mathrm{R}$ used to represent these values for the two side holes, $p_{2}$ and $p_{3}$. The minimum pressures for the bottom hole, $p_{4}$, in the negative and positive yaw angles are presented by $B$ in Figure 4(c). They occur at the yaw angles of -35 and 40 degrees and have almost the same magnitude.

For the present four-hole probe, $p_{i}$ is not maximum for the holes in the yaw plane $\left(P_{1}, P_{2}\right.$, and $\left.P_{3}\right)$ at a pitch angle of 0 degrees (Figure $4(\mathrm{~d})$ ). Instead, $p_{1}$ is maximum at a pitch angle of -10 degrees and at a yaw angle of 0 degrees, $p_{2}$ is maximum 


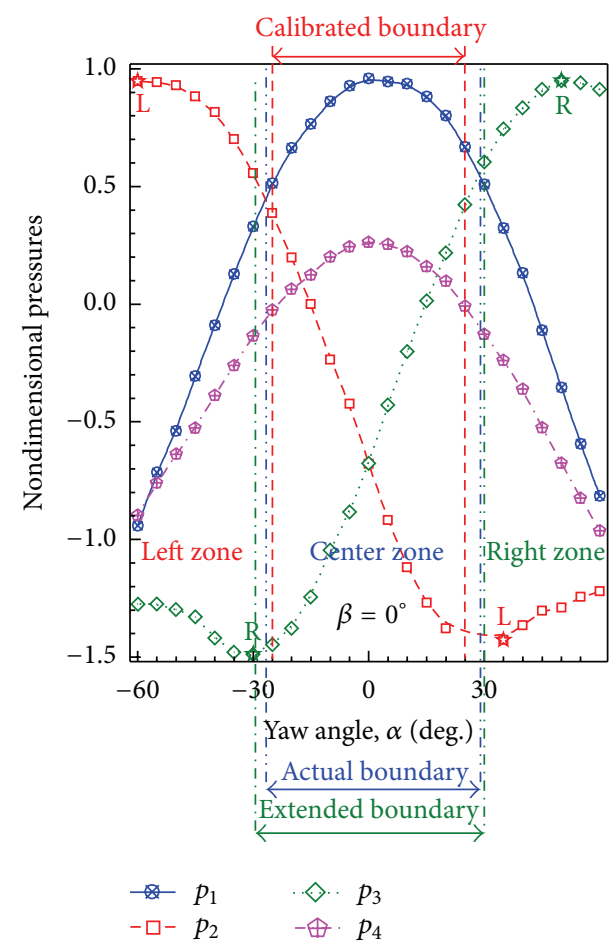

(a)

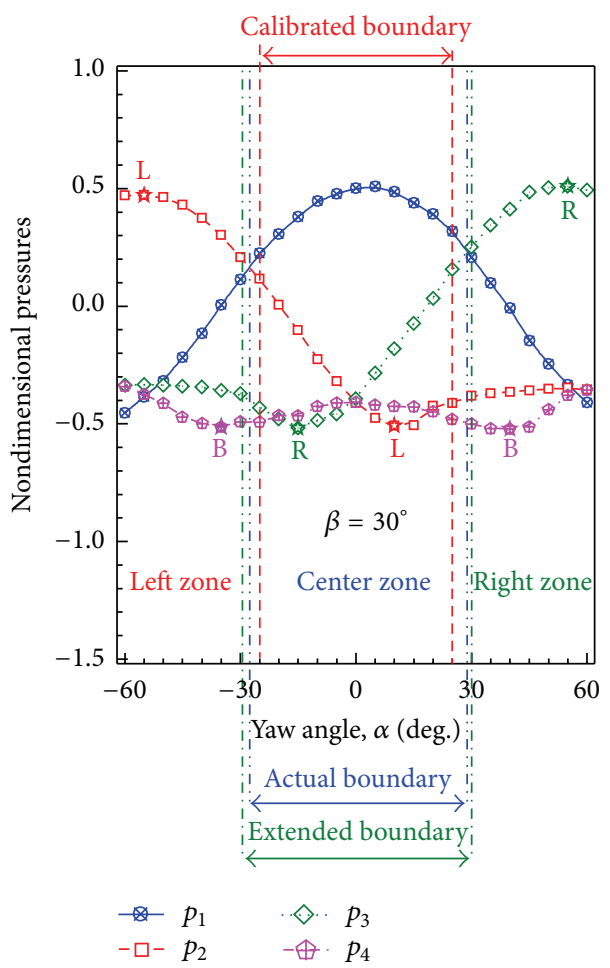

(c)

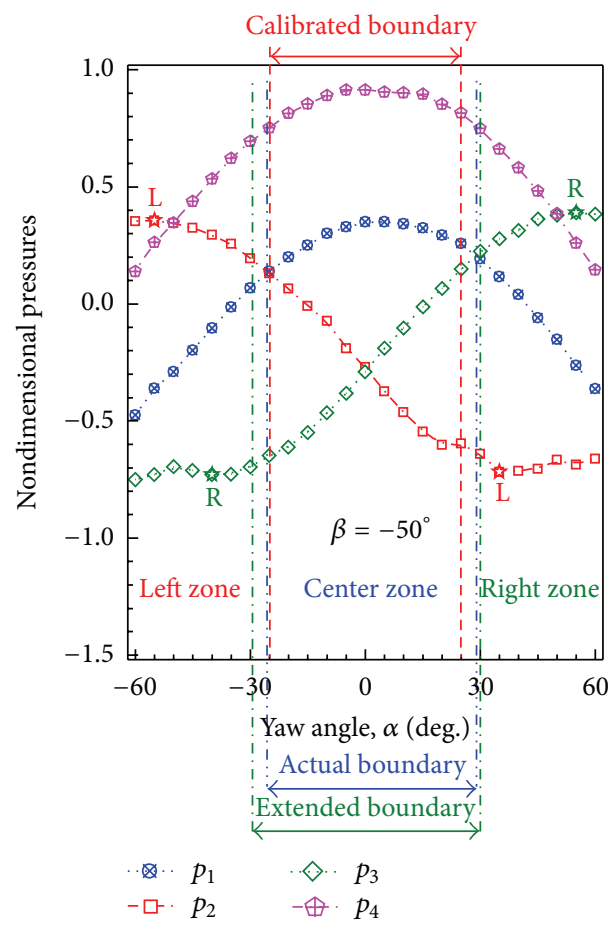

(b)

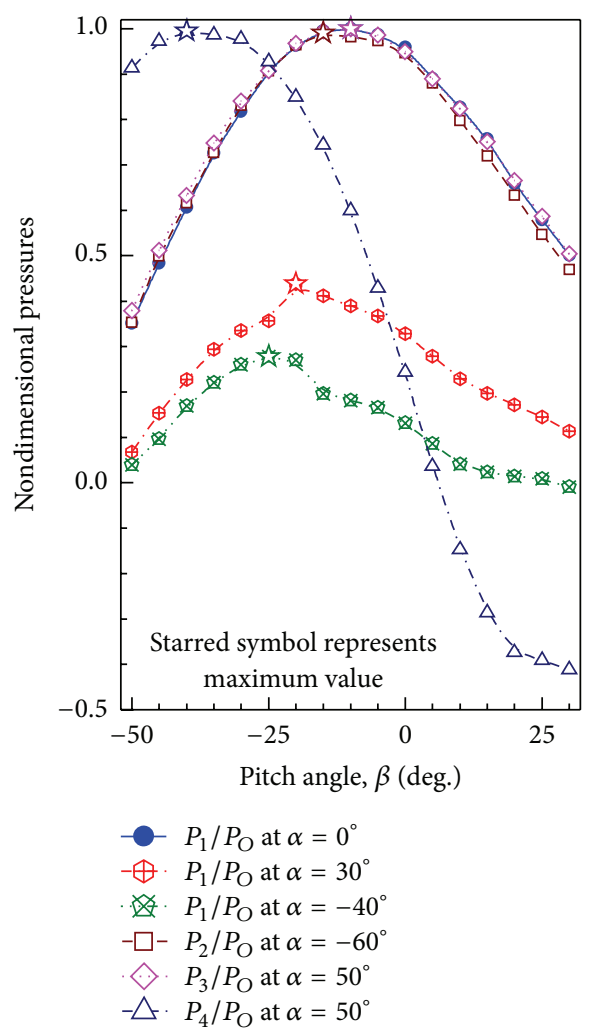

(d)

FIgURE 4: Pressure distributions of the four-hole probe and zone division. 


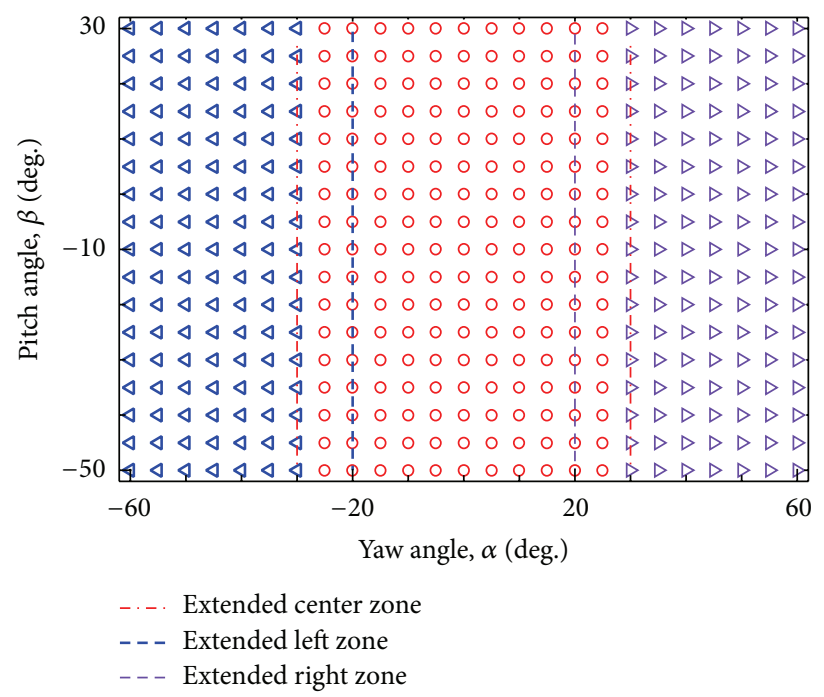

\begin{tabular}{lcccc}
\hline & J Left zone & Center zone & Right zone & Total \\
\hline Number of calibration points & 119 & 187 & 119 & 425 \\
Percentage & 28 & 44 & 28 & 100 \\
Yaw angle range & $-60^{\circ}$ to $-30^{\circ}$ & $-25^{\circ}$ to $25^{\circ}$ & $30^{\circ}$ to $60^{\circ}$ \\
Number of points in extended zone & 153 & 221 & 153 \\
Yaw angle range in extended zone & $-60^{\circ}$ to $-20^{\circ}$ & $-30^{\circ}$ to $30^{\circ}$ & $20^{\circ}$ to $60^{\circ}$ \\
\hline
\end{tabular}

FIGURE 5: Zone division including extended zones.

at a pitch angle of -15 degrees and a yaw angle of -60 degrees, and $p_{3}$ is maximum at a pitch angle of -10 degrees and a yaw angle of 50 degrees. The shift in the maximum pressure coefficient from 0 degrees in pitch angle to $-10 /-15$ degrees in pitch angle is due to the placing of the three holes in yaw plane at the leading edge of the hemispherical shaped head. At each pitch angle, the pressure coefficients $p_{2}, p_{3}$ are similar to $p_{1}$ except that their distributions are shifted by the construction angle. The pressure coefficient of the bottom hole, $p_{4}$, is maximum when the pitch angle is -40 degrees (Figure $4(\mathrm{~d})$ ) and reduces as the pitch angle becomes positive. At a pitch angle of +30 degrees, the pressure coefficient, $p_{4}$, almost becomes constant over the entire yaw angle range (Figure $4(\mathrm{c})$ ). This is one of the reasons why the probe cannot be used beyond +30 degrees in pitch angle. At this pitch angle, the bottom hole is at an angle of 45 degrees with respect to the flow direction and the flow is prone to separation at the bottom hole tap. However, the pitch range of the probe in positive pitch direction can be further increased by reducing the construction angle, $\delta_{2}$. Argüelles Díaz et al. [11] had shown that a construction angle of 25 degrees gave a larger yaw angle range for a three-hole probe. Sitaram and Srikanth [16] had experimentally verified that a smaller construction angle increases the calibration range of a five-hole probe in both the yaw and the pitch planes.

Figure 5 shows the division of the different zones over the entire calibration range. The zones are identified using the pressure measured in the holes of the yaw plane $\left(P_{1}\right.$,
$P_{2}$, and $P_{3}$ ). The division of each zone is based upon the angular interval where one of the pressures in the holes of the yaw plane is maximum. As a result, there are three zones: center zone, when $P_{1}$ is the highest pressure, left zone, when $P_{2}$ is the highest pressure, and right zone, when $P_{3}$ is the highest value. As indicated in Figure 4, the center zone has a boundary of \pm 25 degrees. However, this is not the real boundary of the center zone; it is the calibrated boundary. This arises as the calibration is carried out over intervals of 5 degrees. The actual boundary of the zones is identified using the criterion of equal pressures from the probe holes in the adjacent zones. The boundary between the center and left zones is identified at the yaw angle, where $p_{1}=p_{2}$. The boundary between the center and right zones is identified at the yaw angle, where $p_{1}=p_{3}$. The actual boundary of center zone, left zone, and right zones always falls in between \pm 25 degrees and \pm 30 degrees (see Figures 4(a), 4(b), and $4(\mathrm{c})$ ). Sometimes a measured data point may fall in between these two boundaries, say $\alpha=27.5$ degrees. Although it is possible to predict to which zone they belong (center zone or right zone, depending upon which pressure, center, or right hole is maximum), local calibration data are not available. In such cases, there are two methods of determining the calibration coefficients: extrapolation or interpolation with a zone extension [11]. Extrapolation is not recommended because it may result in larger errors. In the present case, interpolation with a zone extension is used. Zone extension is carried out for each zone to the nearest surrounding data 
TABLE 1: Definition of calibration coefficients.

\begin{tabular}{|c|c|c|c|c|c|}
\hline Zone & $D$ & $C_{\text {PYAW }}$ & $C_{\text {PPITCH }}$ & $C_{\text {PSTATIC }}$ & $C_{\text {PTOTAL }}$ \\
\hline $\begin{array}{l}\text { Center zone } \\
P_{1} \text { is maximum }\end{array}$ & $P_{1}-\left(P_{2}+P_{3}\right) / 2$ & $\left(P_{2}-P_{3}\right) / D$ & $\left(P_{1}-P_{4}\right) / D$ & {$\left[\left(P_{2}+P_{3}\right) / 2-P_{S}\right] / Q=\left(P_{2}+P_{3}\right) / 2 P_{\mathrm{O}}$} & $\left(P_{\mathrm{O}}-P_{1}\right) / \mathrm{Q}=1-P_{1} / P_{\mathrm{O}}$ \\
\hline $\begin{array}{l}\text { Left zone } \\
P_{2} \text { is maximum }\end{array}$ & $\left(P_{1}+P_{2}\right) / 2-P_{3}$ & $\left(P_{3}-P_{2}\right) / D$ & $\left(P_{1}-P_{4}\right) / D$ & $\left(P_{3}-P_{S}\right) / Q=P_{3} / P_{O}$ & $\left(P_{\mathrm{O}}-P_{2}\right) / \mathrm{Q}=1-P_{2} / P_{\mathrm{O}}$ \\
\hline $\begin{array}{l}\text { Right zone } \\
P_{3} \text { is maximum }\end{array}$ & $\left(P_{1}+P_{3}\right) / 2-P_{2}$ & $\left(P_{3}-P_{2}\right) / D$ & $\left(P_{1}-P_{4}\right) / D$ & $\left(P_{2}-P_{S}\right) / Q=P_{2} / P_{O}$ & $\left(P_{\mathrm{O}}-P_{3}\right) / \mathrm{Q}=1-P_{3} / P_{\mathrm{O}}$ \\
\hline
\end{tabular}

point of the adjacent zone. As a result, the center zone is extended to yaw angles of \pm 35 degrees, and the left and right zones are extended to a yaw angle of -20 and 20 degrees, respectively.

The calibration space is divided into three zones, namely, center (yaw angle range of $\pm 30^{\circ}$ ), left (yaw angle range of $-60^{\circ}$ to $-20^{\circ}$ ), and right (yaw angle range of $20^{\circ}$ to $60^{\circ}$ ) zones, with zones overlapping so that no yaw region is left without calibration coefficients. Calibration coefficients are defined for each zone and computed. The calibration coefficients for each zone are defined as shown in Table 1.

In Table $1, Q=P_{O}-P_{S}=P_{O}\left(Q \rightarrow P_{O}\right.$ as $\left.P_{S} \rightarrow 0\right)$.

The calibration curves for the center, left, and right zones are shown in Figures 6 and 7. The calibration curves of the left zone are almost a mirror image of the calibration curves of the right zone. Although the calibration was carried out over intervals of 5 degrees, for the sake of clarity, the calibration curves are presented for intervals of 10 degrees. Calibration curves of $C_{\text {PYAW }}$ versus $C_{\text {PPITCH }}$ are presented as grids, while $C_{\text {PTOTAL }}$ and $C_{\text {PSTATIC }}$ are presented as contours with $C_{\mathrm{PYAW}}$ and $C_{\mathrm{PPITCH}}$ on the $x$ and $y$ axes. The calibration curves of $C_{\text {PYAW versus }} C_{\text {PPITCH }}$ for all three zones are represented by spline curves passing through each calibration data point. In the calibration curves of $C_{\mathrm{PYAW}}$ versus $C_{\mathrm{PPITCH}}$, nearly vertical lines are at constant yaw angle, $\alpha$, and nearly horizontal curves are at constant pitch angle, $\beta$. In an ideal case, $C_{\mathrm{PYAW}}$ versus $C_{\mathrm{PPITCH}}$ should be a square over the entire calibration range, but this cannot be achieved in a real situation. However, in the present case each grid seems to be nearly rectangular except at the extremities of yaw and pitch angles in all the zones. At the extremities, the grid is distorted into a diamond shape, especially along the diagonal directions of the center zone.

This severe distortion from a rectangular shape may result in an error of flow angle determination. From the calibration curves of $C_{\text {PYAW }}$ versus $C_{\text {PPITCH }}$ of the center zone, it can be observed that the probe has a smaller range of $C_{\mathrm{PPITCH}}$ values compared to $C_{\text {PYAW }}$ values. This reduced range of $C_{\text {PPITCH }}$ values will result in an increased sensitivity of the probe to small variations of flow in the pitch plane. The reduction in the range of $C_{\text {PPITCH }}$ for the probe is due to employing of only two pressure holes in the pitch plane. The contours of $C_{\text {PTOTAL }}$ and $C_{\text {PSTATIC }}$ seem to be nearly concentric in all three zones.

5.2. Sensitivity Analysis of the Calibration Coefficients. A sensitivity analysis of the calibration coefficients was carried out for the three zones to quantify the accuracy of the measurements. Sensitivity graphs show the variation of the dependent quantities as a function of the independent quantities. The dependent quantities here are the four calibration coefficients, $C_{\mathrm{PYAW}}, C_{\mathrm{PPITCH}}, C_{\mathrm{PTOTAL}}$, and $C_{\mathrm{PSTATIC}}$, and the independent quantities are the yaw angle $(\alpha)$ and the pitch angle $(\beta)$. The sensitivity coefficients are defined as follows:

$$
\Delta C_{\mathrm{P} i}=\frac{C_{\mathrm{P}(i+1)}-C_{\mathrm{P}(i-1)}}{\text { Angle }(i+1)-\text { Angle }(i-1)},
$$

where $C_{\mathrm{P} i}$ refers to one of the four calibration coefficients, namely, $C_{\text {PYAW }}, C_{\text {PPITCH }}, C_{\text {PSTATIC }}$, or $C_{\text {PTOTAL }}$, and Angle is the yaw or pitch angle where the calibration data are taken.

The sensitivity coefficients for the center, left, and right zones are shown in Figures 8 and 9. In the center zone, the probe pressures change rapidly at large yaw and pitch angles. Hence, the calibration coefficients at large values of yaw and pitch angles have higher sensitivity. Higher sensitivity implies more accurate measurements. It is to be kept in mind that small errors in the measured pressures result in large errors in the calibration coefficients and their sensitivity. At low values of the yaw and pitch angles, the sensitivity coefficients are low. The curves in the center zone are almost symmetric with zero value of yaw angle but they are asymmetric with pitch angle; this is due to the asymmetric geometry of the probe holes in the pitch plane. The calibration coefficients in the center zone are less sensitive to the change of the pitch angle than with the yaw angle, a result of having only two holes in the pitch plane, instead of three holes.

5.3. Interpolation Errors. A look up table method has been developed for a five-hole probe by Sitaram and Kumar [17] to determine the four unknown quantities, namely, yaw and pitch angles and static and total pressure coefficients from the calculated yaw and pitch coefficients. A similar method is utilized here for determining the flow quantities from the present four-hole probe configuration. No additional data are taken for interpolation during the calibration of the probe. However, a calibration data interval of 10 degrees, rather than 5 degrees, is used. All the calibration data are directly measured data. A calibration interval of 10 degrees is relatively large. Sumner [18] recommended that this is the largest calibration interval that can be used with a sevenhole probe. The interpolated values are compared with those obtained during calibration. Histograms of errors in yaw and pitch angles are presented in Figure 10. The errors at the 

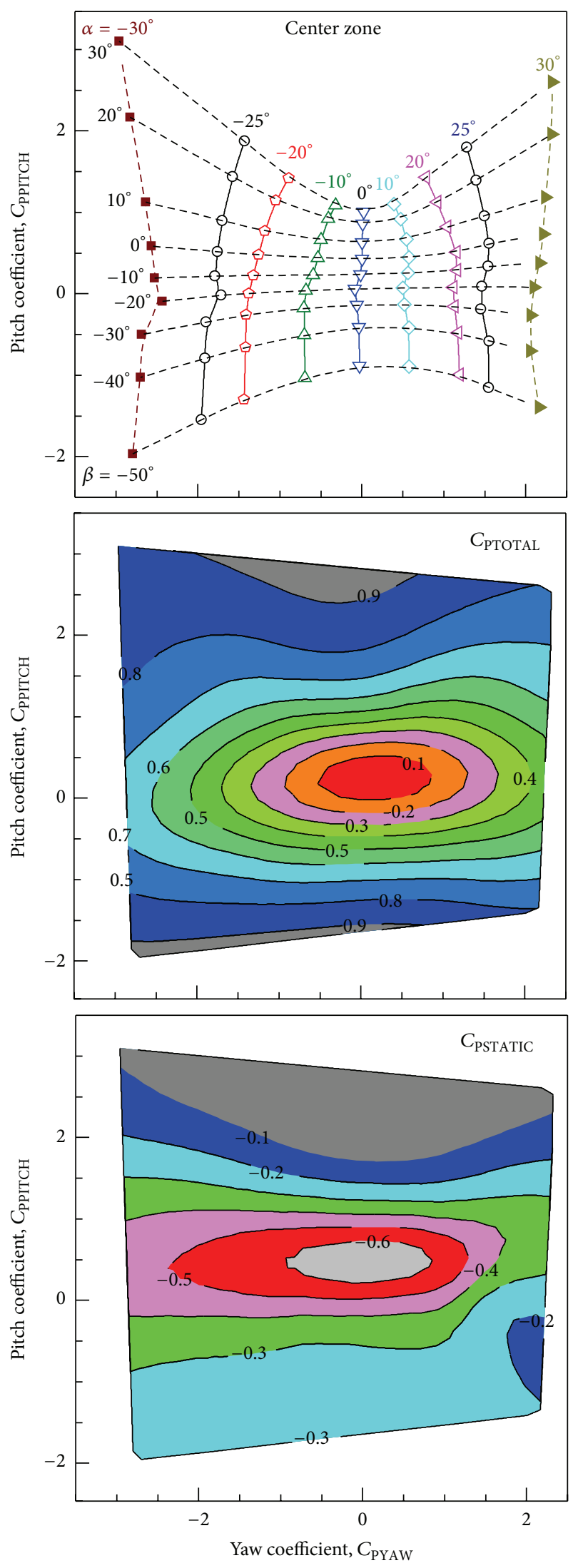

FIgURE 6: Calibration curves of the four-hole probe for the center zone. extremes of the calibration range for each zone are omitted from these figures. For the sake of brevity, histograms of the errors in yaw angle and static pressure coefficients are presented for the central zone and histograms of the errors in pitch angle and total pressure coefficients are presented for the right and left zones, respectively. Most of the errors in yaw and pitch angles are within \pm 1 degrees and most of the errors in total and static and pressures are within -0.01 to $0.02 \%$ of the dynamic head. From the figure, it can be observed that the errors have slightly higher magnitude in the left and right zones compared to the center zone.

The maximum, minimum, RMS, and SD values of errors in yaw and pitch angles and total, static, and dynamic pressures are also presented in Table 2.

Except for yaw and pitch angles and the static pressure coefficient in the right zone, the errors are very small. The large values of errors occur near the extreme range of the calibration zones. The errors are due to the data reduction program only. All other measurement errors such as instrumentation errors, errors due to the calibration (zero angle settings, pitch and yaw angle measurements during calibration, etc.), are not included. For Table 2, the calibration data is given at an interval of 10 degrees. The calibration data at an interval of 5 degrees (excluding the data at 10-degree interval) are given as measured data. The errors are almost negligible when both calibration data and measured data are given at an interval of 5 degrees. The errors presented by Lee and Jun [19], who used a calibration interval of 5 degrees in their data reduction program, have similar magnitude.

\section{Conclusions}

From the present investigation, the following major conclusions are drawn:

(1) The calibration range of a cantilever type four-hole probe is extended to \pm 60 degrees in the yaw plane and -50 to +30 degrees in the pitch plane. This is achieved by dividing the calibration space into three zones, namely, center, left, and right zones. The zones are overlapping so that no point in the calibration space is left without calibration coefficients. In each of the zones, the calibration coefficients are defined differently.

(2) The probe pitch sensitivity is lower than the yaw sensitivity in the center zone. Extended left and right zones have lower sensitivity than the center zone.

(3) Errors due to the data reduction program for the probe are presented for all the zones and the errors are found to be reasonably low in all three zones. However the errors in the extended left and right zones have slightly larger magnitudes compared to those in the center zone.

(4) From the present investigation, it can be concluded that the probe can be used for measurement of highly three-dimensional flows that occur in turbomachinery and other aerodynamic flows, particularly in confined measurement spaces. 

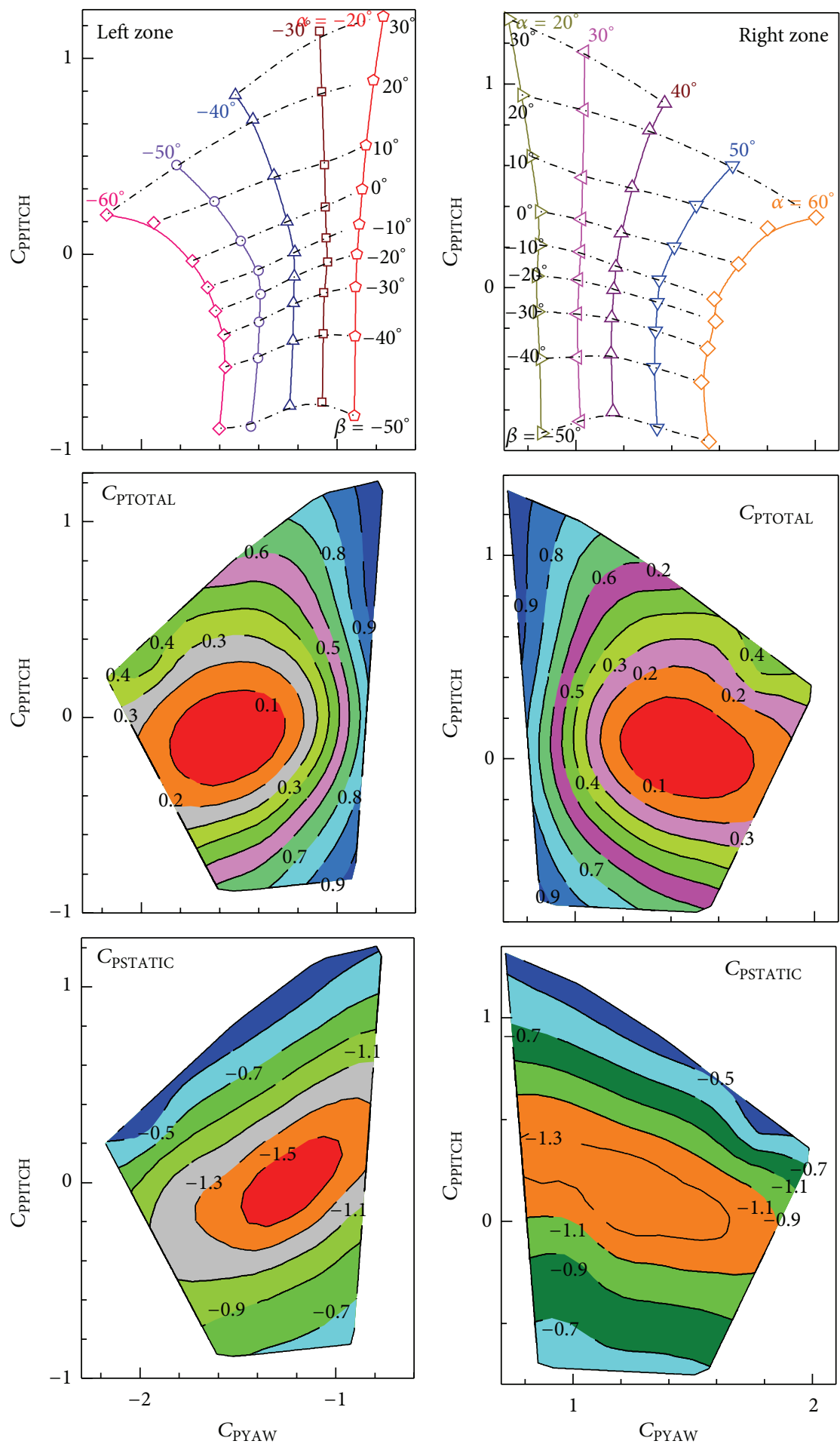

Figure 7: Calibration curves of the four-hole probe for the left and right zones.

(5) It is important to emphasize that measurements by multihole probes are affected by flow Mach and Reynolds numbers. Hence it is essential that the probes be calibrated at different Mach and Reynolds numbers and methods be developed to account for these effects on the probe measurements. The calibration is presented at only one velocity to establish the extended calibration technique. The probe will be calibrated at different Mach and Reynolds numbers and methods will be developed to include these effects 

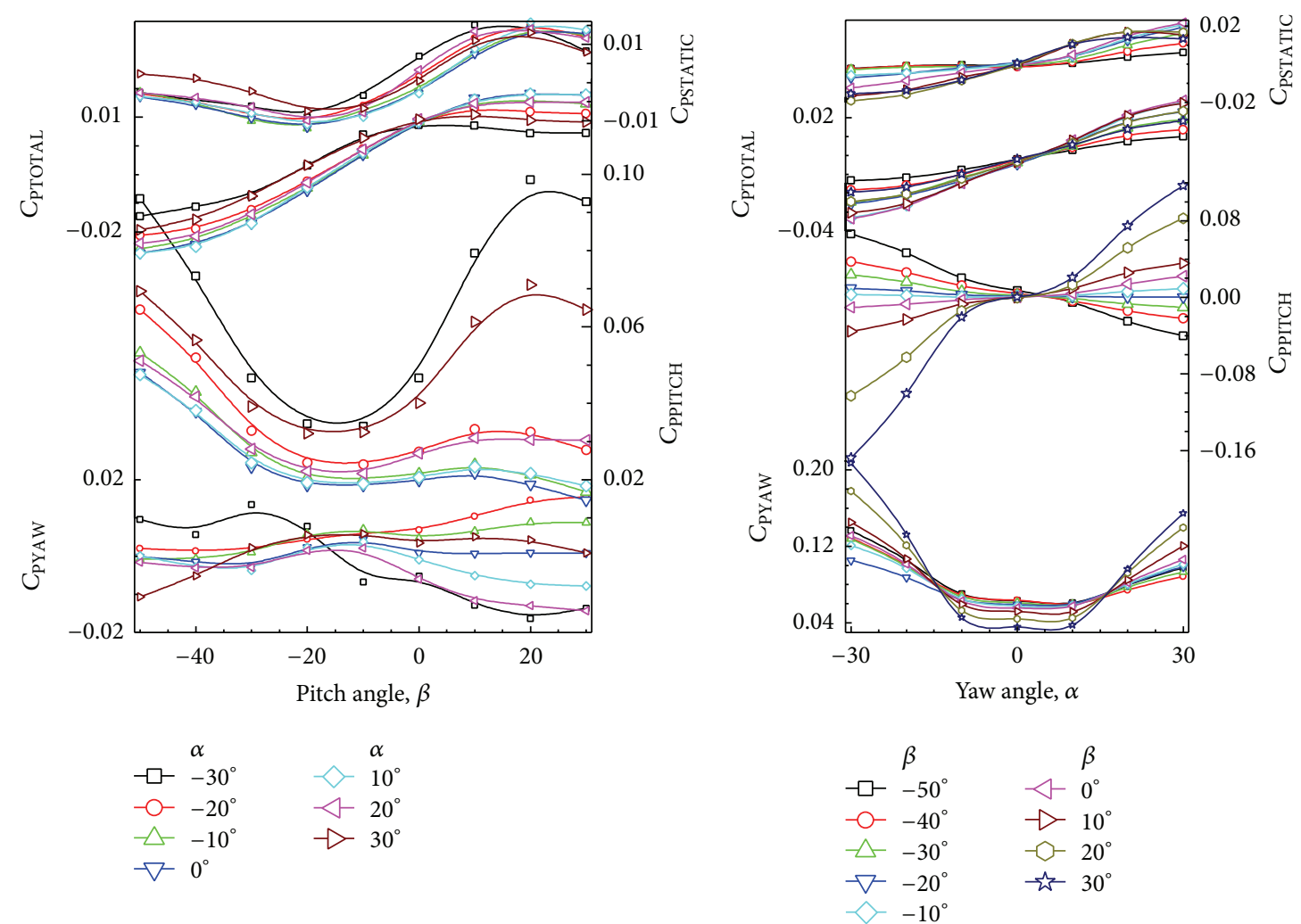

FIGURE 8: Sensitivity curves of the calibration coefficients in the center zone.
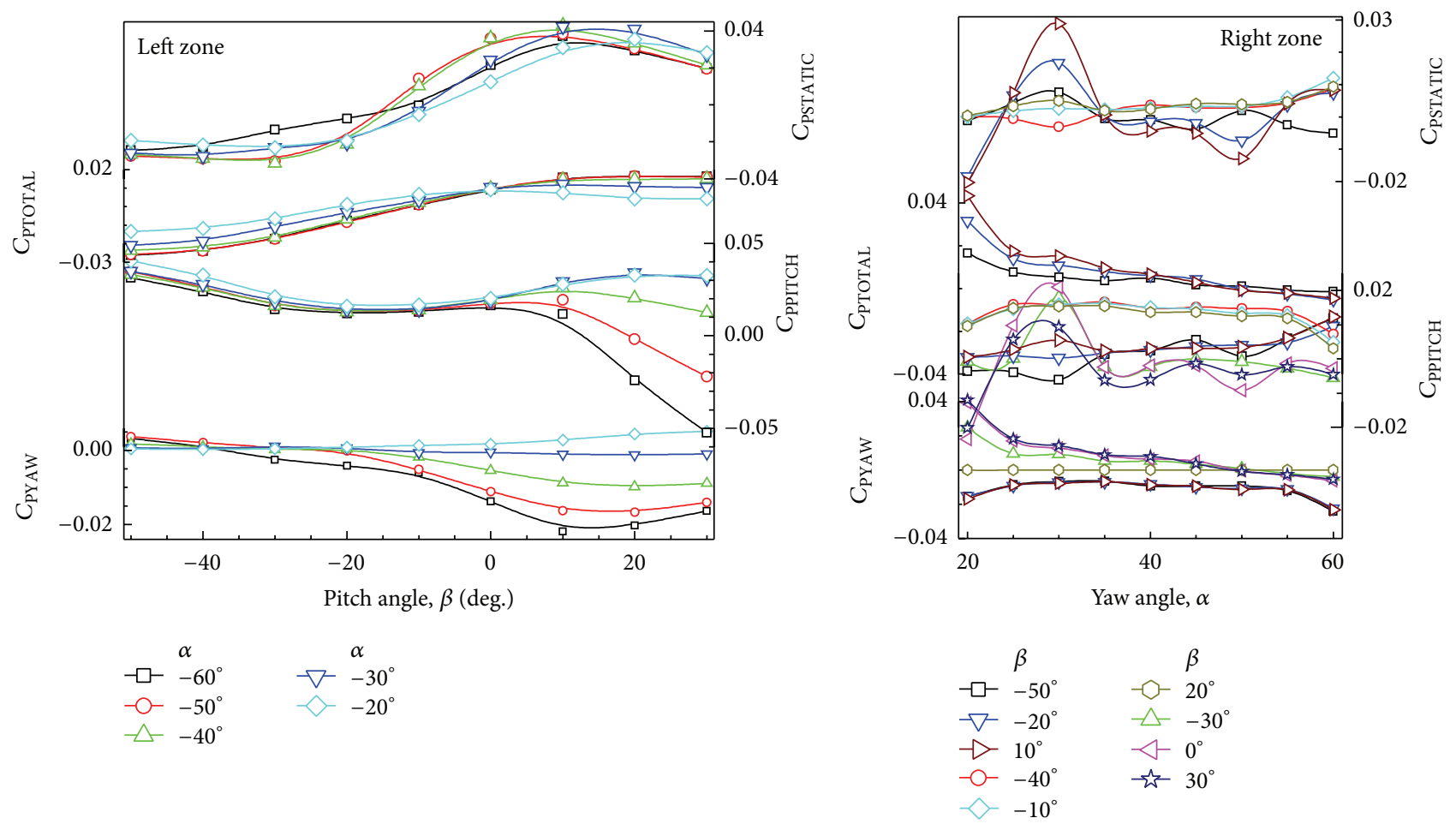

FIGURE 9: Sensitivity curves of the calibration coefficients in the left and right zones. 

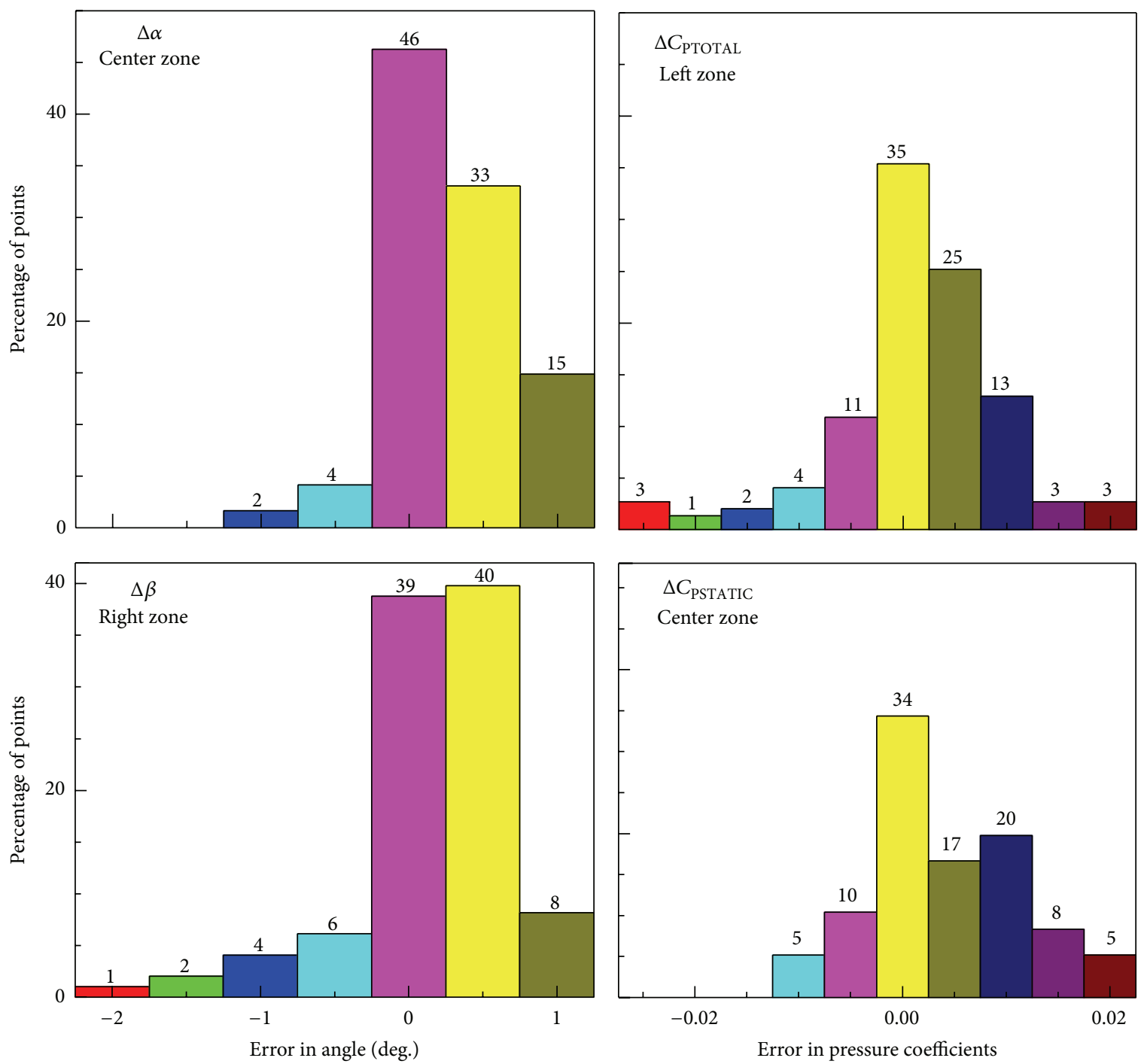

FIGURE 10: Histograms of interpolation errors of the four-hole probe in different zones.

TABLE 2: Errors in yaw and pitch angles and total and static pressure coefficients.

\begin{tabular}{|c|c|c|c|c|c|c|c|c|}
\hline \multirow[t]{2}{*}{ Zone } & \multicolumn{4}{|c|}{ Error in yaw angle, $\Delta \alpha$ (deg.) } & \multicolumn{4}{|c|}{$\begin{array}{l}\text { Error in total pressure coefficient, } \\
\qquad \Delta C_{\text {PTOTAL }}\end{array}$} \\
\hline & Max & Min & RMS & SD & Max & Min & RMS & SD \\
\hline Center & 1.00 & -1.27 & 0.36 & 0.35 & 0.020 & -0.029 & 0.012 & 0.008 \\
\hline Left & 0.85 & -1.27 & 0.32 & 0.32 & 0.020 & -0.029 & 0.008 & 0.008 \\
\hline Right & 0.61 & -2.03 & 0.54 & 0.54 & 0.022 & -0.015 & 0.008 & 0.008 \\
\hline \multirow[t]{2}{*}{ Zone } & \multicolumn{4}{|c|}{ Error in pitch angle, $\Delta \beta$ (deg.) } & \multicolumn{4}{|c|}{$\begin{array}{c}\text { Error in static pressure coefficient, } \\
\Delta C_{\mathrm{PSTATIC}}\end{array}$} \\
\hline & Max & Min & RMS & SD & $\operatorname{Max}$ & Min & RMS & SD \\
\hline Center & 0.89 & -1.34 & 0.59 & 0.38 & 0.028 & -0.028 & 0.011 & 0.010 \\
\hline Left & 0.89 & -1.14 & 0.33 & 0.33 & 0.028 & -0.028 & 0.010 & 0.010 \\
\hline Right & 0.85 & -2.11 & 0.53 & 0.50 & 0.027 & -0.039 & 0.015 & 0.014 \\
\hline
\end{tabular}


on the probe measurements. These results will be presented in a future paper.

\section{Nomenclature}

Center, left, Center, left, and right zones of calibration and right: space (see Figures 4 and 5)

$C_{\text {PPITCH }}$ : Pitch coefficient (defined in text)

$C_{\text {PSTATIC }}$ Static pressure coefficient (defined in text)

$C_{\text {PTOTAL }}$ : Total pressure coefficient (defined in text)

$C_{\text {PYAW }}$ : $\quad$ Yaw coefficient (defined in text)

D:

Max, Min, Maximum, minimum, root mean square,

RMS, and and standard deviation values of

SD: interpolation error

$P_{O}$ : Total pressure, $\mathrm{Pa}$

$P_{S}$ : $\quad$ Static pressure, $\mathrm{Pa}$

$P_{1}, P_{2}, P_{3}$, Pressures measured by probe holes 1 to 4 , and $P_{4}$ :

$p_{1}, p_{2}, p_{3}$, and $p_{4}$ : $\mathrm{Pa}$

Pressures measured by probe holes 1 to 4 , nondimensionalized with $P_{O}$

Q: Dynamic pressure $=P_{O}-P_{S}=P_{O}$ $\left(Q \rightarrow P_{O}\right.$ as $\left.P_{S} \rightarrow 0\right), \mathrm{Pa}$

$\alpha: \quad$ Yaw angle, deg.

$\beta$ : $\quad$ Pitch angle, deg.

$\Delta \alpha: \quad$ Interpolation error of yaw angle, deg.

$\Delta \beta: \quad$ Interpolation error of pitch angle, deg.

$\Delta C_{\mathrm{P}}: \quad$ Sensitivity of calibration coefficient, $C_{\mathrm{P}}$

$\Delta C_{\text {PSTATIC }}$ Interpolation error of static pressure coefficient

$\Delta C_{\text {PTOTAL }}:$ Interpolation error of total pressure coefficient.

\section{Competing Interests}

The authors declare that there is no conflict of interests regarding the publication of this paper.

\section{References}

[1] N. Sitaram, B. Lakshminarayana, and A. Ravindranath, "Conventional probes for the relative flow measurement in a turbomachinery rotor blade passage," ASME Journal of Engineering for Power, vol. 103, no. 2, pp. 406-414, 1981.

[2] D. Telionis, Y. Yang, and O. Rediniotis, "Recent developments in multi-hole probe technology," in Proceedings of the 20th International Congress of Mechanical Engineering (COBEM '09), Gramado, Brazil, November 2009.

[3] T. J. Dudzinski and L. N. Krause, "Flow-direction measurement with fixed-position probes," Tech. Rep. NASA-TM-X-1904, 1969.

[4] N. Sitaram and A. L. Treaster, "A simplified method of using four-hole probes to measure three-dimensional flow fields," Journal of Fluids Engineering, vol. 107, no. 1, pp. 31-35, 1985.

[5] A. L. Treaster and A. M. Yocum, "The calibration and application of five-hole probes," ISA Transactions, vol. 18, no. 3, pp. 2334, 1979.
[6] R. W. Gallington, "Measurement of very large flow angles with non-nulling seven-hole probes," Tech. Rep. USAFA-TR-80-17, 1980.

[7] A. J. Pisasale and N. A. Ahmed, "A novel method for extending the calibration range of five-hole probe for highly threedimensional flows," Flow Measurement and Instrumentation, vol. 13, no. 1-2, pp. 23-30, 2002.

[8] C. Ostowari and W. H. Wentz Jr., "Modified calibration technique of a five-hole probe for high flow angles," Experiments in Fluids, vol. 1, no. 3, p. 166, 1983.

[9] N. Sitaram and M. Govardhan, "Large angle calibration of five hole probes," Journal of the Aeronautical Society of India, vol. 54, no. 3, pp. 265-272, 2002.

[10] C. Venkateswara Babu, M. Govardhan, and N. Sitaram, "A method of calibration of a seven-hole pressure probe for measuring highly three-dimensional flows," Measurement Science and Technology, vol. 9, no. 3, pp. 468-476, 1998.

[11] K. M. Argüelles Díaz, J. M. Fernández Oro, and E. Blanco Marigorta, "Direct calibration framework of triple-hole pressure probes for incompressible flow," Measurement Science and Technology, vol. 19, no. 7, Article ID 075401, 2008.

[12] K. M. Argüelles Díaz, J. M. Fernández Oro, and E. Blanco Marigorta, "Extended angular range of a three-hole cobra pressure probe for incompressible flow," ASME Journal of Fluids Engineering, vol. 130, no. 10, Article ID 101401, pp. 1-6, 2008.

[13] I. C. Shepherd, "A four-hole pressure probe for fluid flow measurements in three dimensions," Journal of Fluids Engineering, vol. 103, no. 4, pp. 590-594, 1981.

[14] J. P. Schlienger, Evolution of unsteady secondary flows in a multistage shrouded axial turbine, ETH no. 15230 [Ph.D. dissertation], ETH, Zurich, Switzerland, 2003.

[15] D. Contini, G. Manfrida, and V. Michelassi, "Secondary flow measurements in a gas turbine cascade by a 3D pneumatic probe," in Proceedings of the 14th Symposium on Measuring Techniques for Transonic and Supersonic Flows in Cascades and Turbomachines, Limerick, Ireland, 1999.

[16] N. Sitaram and K. Srikanth, "Effect of chamfer angle on the calibration curves of five hole probes," International Journal of Rotating Machinery, vol. 2014, Article ID 704315, 11 pages, 2014.

[17] N. Sitaram and S. Kumar, "Look up table method for five hole probe data reduction," in Proceedings of the 38th National Conference on Fluid Mechanics and Fluid Power, Paper no. EM0, 8 pages, Bhopal, India, December 2011.

[18] D. Sumner, "A comparison of data-reduction methods for a seven-hole probe," Journal of Fluids Engineering, vol. 124, no. 2, pp. 523-527, 2002.

[19] S. W. Lee and S. B. Jun, "Reynolds number effects on the non-nulling calibration of a cone-type five-hole probe for turbomachinery applications," Journal of Mechanical Science and Technology, vol. 19, no. 8, pp. 1632-1648, 2005. 


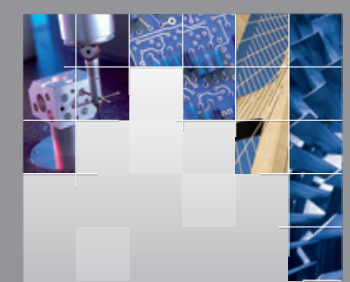

\section{Enfincering}
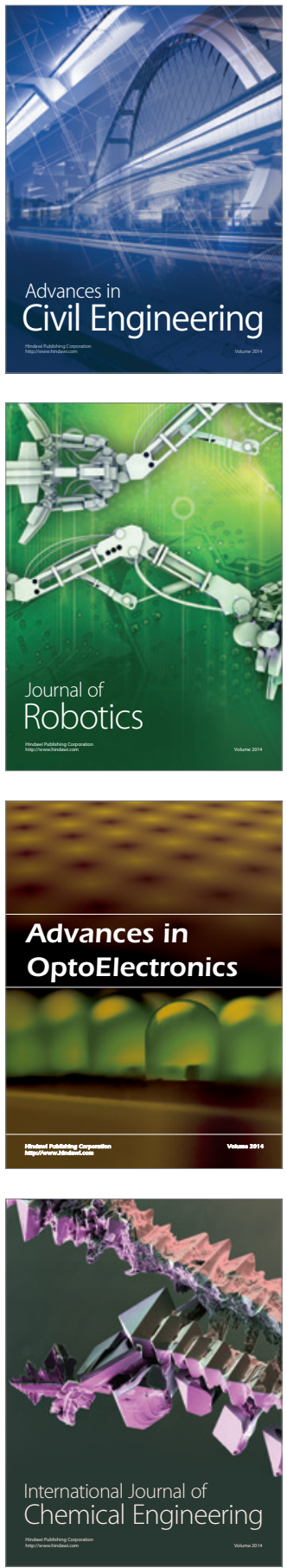

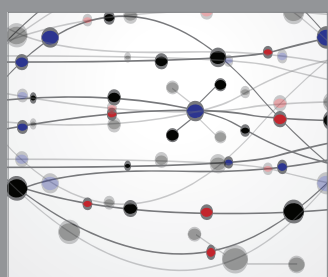

The Scientific World Journal

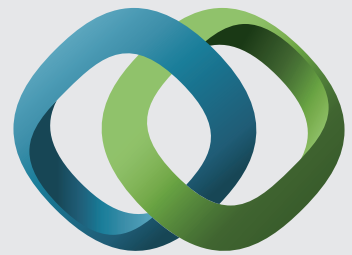

\section{Hindawi}

Submit your manuscripts at

http://www.hindawi.com
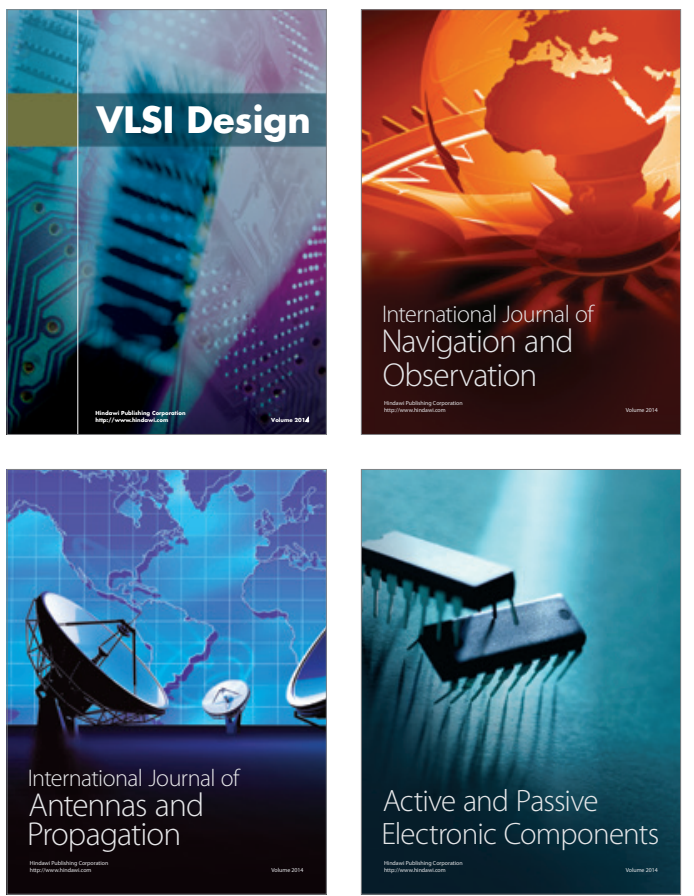
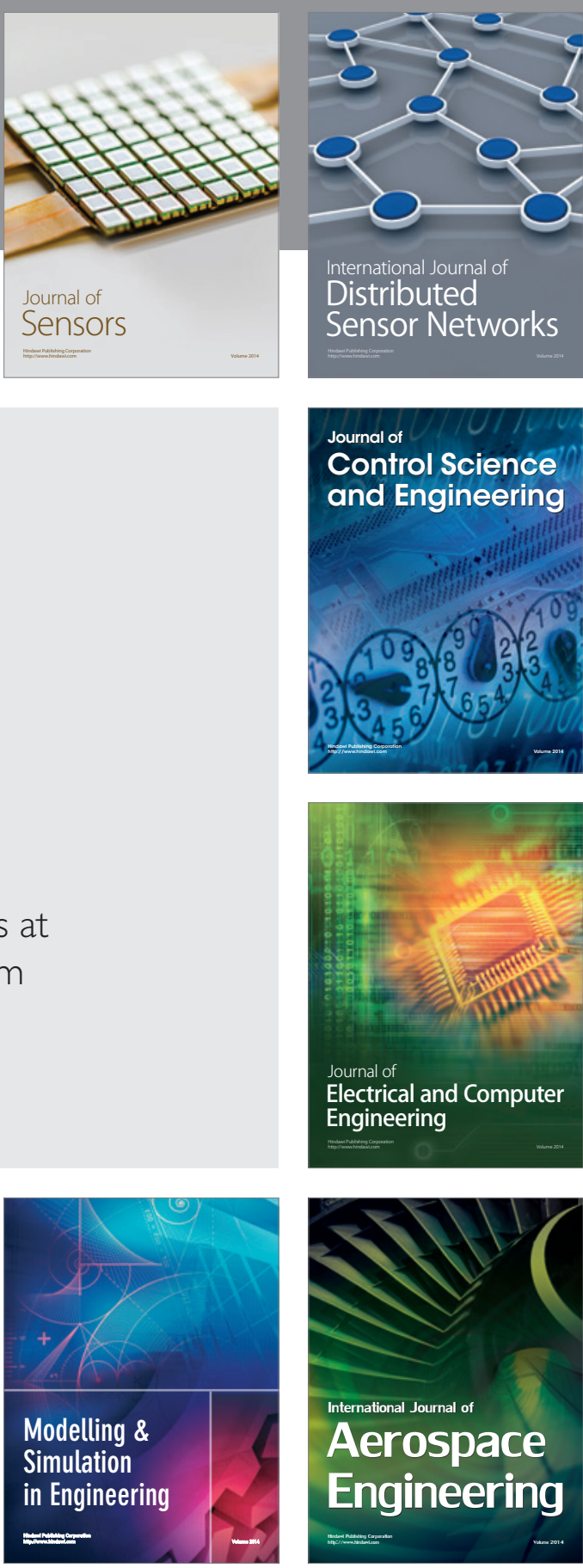

International Journal of

Distributed

Sensor Networks

Journal of

Control Science

and Engineering
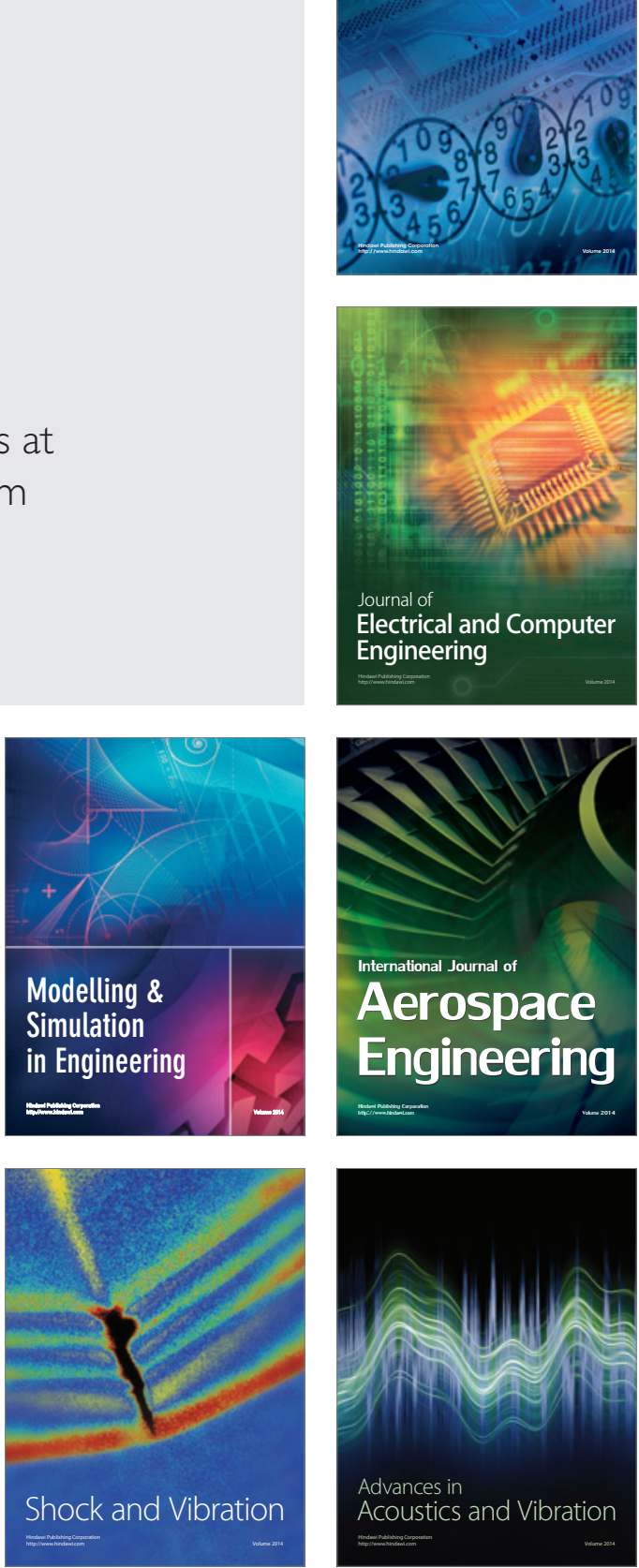